\title{
Ultrapulsed CO2 Laser Combined with Long-pulsed Nd: YAG Laser for the Treatment of Oral and Maxillofacial Pyogenic Granuloma
}

\section{Licheng Jiang}

Liaocheng People's Hospital

Di Zhang

Liaocheng People's Hospital

Keyi Li

Liaocheng People's Hospital

Bin Zhang

Liaocheng People's Hospital

Anqi Zhang ( $\square$ zhang_angela1119@163.com )

Liaocheng People's Hospital https://orcid.org/0000-0001-5128-2464

Jianlin Liu

Liaocheng People's Hospital

Research article

Keywords: pyogenic granuloma, oral and maxillofacial region, ultrapulsed CO2 laser, long-pulsed Nd: YAG laser

Posted Date: August 25th, 2020

DOI: https://doi.org/10.21203/rs.3.rs-51199/v1

License: (c) (1) This work is licensed under a Creative Commons Attribution 4.0 International License.

Read Full License 


\section{Abstract}

Background: Pyogenic granuloma is a common, benign, acquired vascular lesion involving the skin and mucous membrane. It occurs in exposed areas, such as oral and maxillofacial regions, hands and feet, which usually have aesthetic affections on the patients' appearance. Although pyogenic granuloma is treatable by a variety of treatments, they bear defects such as high recurrence rate, multiple therapies, leaving obvious scars, hyperpigmentation, or skin atrophy. In this study, we treat pyogenic granuloma patients with both ultrapulsed $\mathrm{CO}_{2}$ laser and long-pulsed $\mathrm{Nd}$ : YAG laser, in order to achieve ideal effects.

Methods: 20 participants with pyogenic granuloma of the oral and maxillofacial region were treated with ultrapulsed $\mathrm{CO}_{2}$ laser and long-pulsed Nd: YAG laser, reexamined one month later, and followed up for one year. Clinical characteristics and treatment effects were recorded and examined.

Results: The 20 participants were all cured by one treatment with a cure rate of $100 \%$. None of the patients had scar or recurrence during12 months of follow-up.

Conclusions: Ultrapulsed $\mathrm{CO}_{2}$ laser combined with long-pulsed Nd: YAG laser is a safe and cosmetically effective solution for pyogenic granuloma of the oral and maxillofacial region.

Trial registration: This research is a retrospective study.

\section{Background}

Pyogenic granuloma (PG), also known as lobular capillary hemangioma, occurs in the face, neck, and upper limbs at any age, mostly single-shot ${ }^{[1]}$. The lesion usually starts as a red spot of pinhole size. With repeated mild irritation, immune damage of the patient, pregnancy, or antiretroviral drugs, the spot forms rapidly growing, easily broken red papule or polyp ${ }^{[2-4]}$. It overgrows rapidly in weeks or months, then forms a soft and elastic hemisphere with a smooth red surface, which is easily ruptured and bleeding after wound. The patients generally have no symptoms except occasionally erosion and exudation. However, since PG usually occurs in the exposed area and does not resolve without treatment, most patients visit the clinic for cosmetic reason and few for bleeding or pain.

Various clinical treatments have been developed for PG, including surgery, cryotherapy, laser ablation, and drug therapy such as $\beta$-adrenergic blockers and sclerotherapy ${ }^{[5-7]}$. In the present study, ultrapulsed $\mathrm{CO}_{2}$ laser and long-pulsed Nd: YAG laser were applied in combination in the treatment of oral and maxillofacial PG. One session of treatment achieved satisfying outcomes with a $100 \%$ cure rate and leaving no visible scar, indicating that the combined ultrapulsed $\mathrm{CO}_{2}$ laser and long-pulsed Nd: YAG laser is an ideal treatment for PG.

\section{Methods}


Our research subjects were enrolled from the Department of Oral and Maxillofacial Surgery in Liaocheng People's Hospital from October 2015 to October 2018. Inclusion criteria: the patients were diagnosed as PG by two maxillofacial surgeons according to medical history, clinical manifestation and ultrasonography. Exclusion criteria: the patients had local infection around lesion; had severe liver or kidney dysfunction; implanted with a cardiac pacemaker; had hemorrhagic or coagulopathy diseases, or were taking anticoagulant; were pregnant or lactating; were unable to comply with the requirements of postoperative avoiding water or sunscreen; had a history of epilepsy or psychological disorders. This study was approved by the Ethics Committee of Liaocheng People's Hospital, and the informed consent forms were obtained from each patient or their families. Clinical information and medical history of the patients was collected before treatment.

The ultrapulsed $\mathrm{CO}_{2}$ laser (Miracle, China, a wavelength of $10,600 \mathrm{~nm}$ and pulse width of $<400 \mathrm{us}$ ) and long-pulsed Nd: YAG laser (FOTONA, a wavelength of 1,064 nm) were applied in combination in our research.

Each lesion was photographed with the same camera parameters in a specific studio before the operation and at each subsequent visit. Photos were archived and the curative effect was evaluated at each visit. Patients and operators were protected by special goggles with filters. The treatment area was exposed and routinely disinfected 3 times by $1 \%$ benzalkonium bromide, and local infiltration anesthesia was conducted by mepivacaine hydrochloride $(20 \mathrm{mg} / \mathrm{ml})$ and adrenaline injection $(0.01 \mathrm{mg} / \mathrm{ml})$ under the lesions. A sterilized cotton swab was placed at the root of the lesion, and the granuloma was forced to a pale ischemic state.

The $\mathrm{CO}_{2}$ laser energy used for operation ranged from 2 3 W; bleeding or large PG was operated with a higher power (2.5 3 W). The ultrapulsed $\mathrm{CO}_{2}$ laser beam was focused and directed at lesion root for carbonization and vaporization, and the lesion was separated from the root. Then under the 50-fold magnifying glass, the lesion basis was vaporized and cleaned layer by layer. During cleaning, the evaporated residue was wiped off with a cotton swab dipped with physiological saline to ensure complete removal of the granuloma tissue. By the end of $\mathrm{CO}_{2}$ laser ablation, the lesion was completely removed, and the base was flat without bleeding. The long-pulsed Nd: YAG laser was then used to irradiate the lesion area vertically, covering the base part, with a spot size of $2 \mathrm{~mm}$, a pulse width of 5-20 ms and energy of 80-100 J/cm². No gray was observed after irradiation.

After operation, erythromycin ointment was applied to the treated area. Hydrocolloid dressing (algoplaque) was appropriately trimmed and referred to the treatment area, and ice compress was applied for 30 minutes immediately to prevent postoperative skin heat damage. Erythromycin ointment and algoplaque were replaced once every 2-3 days for 4 weeks. The patients visited the clinic 4 weeks after treatment, and the next treatment was planned according to healing and recovery. Patients were followed up to 12 months after the operation.

\section{Results}




\section{Demographics and lesion features}

20 patients were involved in this study, including 13 males and 7 females, with a male to female ratio of 1.86:1. The average age was $12.85 \pm 16.18$, ranged from 1 to 61 , and $85 \%$ of patients were juveniles ( $<18$ years old).

The courses of disease ranged from 1 week to 6 months, with an average of 4 weeks. The size of lesions ranged from 2 to $5 \mathrm{~mm}$, with an average of $2.7 \mathrm{~mm}$. 35\% of patients had bleeding; the rests had no clinical symptoms and visited our clinic for aesthetical reason. 55\% oral and maxillofacial PG occurred in the lip, among which $91 \%$ affected lip mucosa. The total mucosal and cutaneous lesions ratio was 1.2:1, which was 2.5:1 in females and 1:1.2 in males. Detailed case information, including affected site and size are listed in Table 1.

\section{Treatment and outcomes}

Treatment parameters, healing time, and adverse effects were listed in Table 2. The results of our study showed that the cure rate of one secession of combined treatment of ultrapulsed $\mathrm{CO}_{2}$ and long-pulsed Nd: YAG laser was $100 \%$. Photos of two representative cases (case A and B, photos of Case B were taken with algoplaque covered) before and after treatment are shown in Figure 1. 55\% patients showed temporary redness, $25 \%$ had temporary pigmentation, $15 \%$ developed ulcers and one patients had blister after treatment; all abovementioned adverse effects resolved spontaneously without special care (Table 2). No obvious scar mark was observed after complete wound healing, and all patients were satisfied with the treatment outcomes. No recurrence happened during the 12 months follow-up.

\section{Discussion}

PG is a red polypoid nodule that is often formed by trauma-caused lobular hyperplasia of the skin, or mucosal capillary and venules. It usually has effects on aesthetics, which was the most common reason for clinic visits ${ }^{[8-10]}$. PG occurs at any age; in this study, most patients were children and adolescents, and males were slightly more than females. The primary sites of oral and maxillofacial PG among our participates were lips, and PG happened more prone in mucosa than skin.

The regular therapies for PG include surgery excision, cryotherapy and $\mathrm{CO}_{2}$ laser ablation. The surgical operation has been wildly used in past decades. The process usually includes resecting pedunculated tissue, and scaling or electrocogulating the residual. Surgery excision has a high cure rate and low recurrence rate, but anesthesia is usually unavoidable; besides, intraoperative bleeding, scarring, pigmentation and hypopigmentation are common, and operation at tricky and cosmetically sensitive sites such as mouth, lip and toe could be difficult. Considering anesthesia-related risks, cryotherapy is safer for PG; it does not cause bleeding during the operation, and scars were rare after healing. However, the contact freezing depth acquires high proficiency, and repeated treatments may be needed to 
thoroughly erase the neoplasm. As a relatively novel therapeutic modality, sclerotherapy showed satisfied clinical effects. The topical application of timolol eye drops and propranolol was effective, and distinct adverse reactions were rarely reported. However, topical treatment is usually lengthy, and the application of $\beta$-adrenergic blockers, especially through oral administration, has certain risks of slowing down the heart rate, causing hypoxemia, hypertension or hyperglycemia ${ }^{[11-13]}$.

Laser therapy has produced successful results in the clinic, with the advantages of minimal pain and elimination for sutures. In the present day, more and more operations were conducted with ultrapulsed $\mathrm{CO}_{2}$ laser. The ultrapulsed $\mathrm{CO}_{2}$ laser has an output wavelength of $10,600 \mathrm{~nm}$, which is mainly absorbed by water in the skin. The generated energy heats the tissue, which destroys lesion by evaporation and ablation, and the thermal effect at dermis also induces cell regeneration to repair the damaged tissue ${ }^{[14]}$. Ultrapulsed $\mathrm{CO}_{2}$ laser produces a fine focusing spot to precisely control the range of treated site and causes less irritation for surrounding tissue, therefore reduces adverse effects such as local edema, hyperpigmentation, hypopigmentation and scar formation, and suitable for operation at tricky sites ${ }^{[15]}$. However, $\mathrm{CO}_{2}$ ablation creates open wounds and therefore has a certain chance of bleeding, recurrence and scaring ${ }^{[5,16,17]}$. Long-pulsed $1,064 \mathrm{~nm} \mathrm{Nd}$ : YAG laser was reported with cosmetically favorable therapeutic outcomes for $\mathrm{PG}^{[18]}$. Its typical features include the deep penetration down to the tissue, thermocoagulation effect for oxyhemoglobin, and contraction function for capillary ${ }^{[19]}$. It can be absorbed by met-hemoglobin and solidifies hemoglobin, which destroys red blood cells and forms

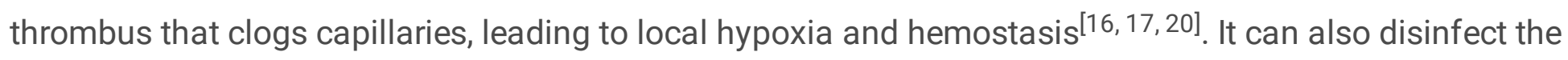
surgical wound with mildest postoperative pain, swelling and pigmentation ${ }^{[21]}$. Despite its advantages, $\mathrm{Nd}$ : YAG penetrates so deeply that not only the capillaries of the granulation tissue, but those beneath lesion might also be solidified, which makes it difficult to judge if the granulation tissue was removed thoroughly. Apart from that, this strategy alone usually requires multiple sessions of treatment for eradication, and may also leave local coloration and depression ${ }^{[2,19,22,23]}$.

In this present study for PG treatment, by combining ultrapulsed $\mathrm{CO}_{2}$ laser and long-pulsed 1,064 nm Nd: YAG laser, we expected to unite their advantages, i.e. the vaporization effects of the former, and the coagulation and vasoconstriction function of the later, thereby reducing the bleeding during the operation and thoroughly removing the lesion with a clear visual field of the operation area, in order to maximize the therapeutic effect and minimize the postoperative complications. According to our follow-up records, all patients (20/20) were cured by one session of combined treatment with no recurrence in 12 months. Notably, PG involved in this study were all located at the oral and maxillofacial areas, which are usually cosmetically sensitive. This combined treatment showed not only a high cure rate, but also mild adverse effects and high satisfaction.

In summary, in the treatment of oral and maxillofacial PG, we firstly combined ultrapulsed $\mathrm{CO}_{2}$ laser with long-pulsed 1,064 nm Nd: YAG laser. With the principle of selective photothermal action, the ultrapulsed $\mathrm{CO}_{2}$ laser and long-pulsed $\mathrm{Nd}$ : YAG laser combined therapeutic strategy can effectively reduce bleeding during operation, improve the accuracy and safety of this medical cosmetic treatment with satisfactory 
outcomes. However, future research with controlled design, larger sample size, longer follow-up and investigation would be helpful to further verify the efficacy of this combined laser treatment.

\section{Abbreviations}

$\mathrm{CO}_{2}$ : Carbon dioxide

Nd:YAG laser: Neodymium-doped yttrium aluminium garnet laser

PG: Pyogenic granuloma

\section{Declarations}

\section{Ethics approval and consent to participate}

This study was approved by the ethics committee of Liaocheng People's Hospital. All patients or parents of the children participated provided written informed consent.

\section{Availability of data and materials}

The datasets used and/or analyzed during the current study are available from the corresponding author upon reasonable request.

\section{Competing interests}

The authors declare that no competing interests exist.

\section{Funding}

This study is supported by the Shandong Province Medical and Health Science Technology Development Plan Project (No.2017WS219 and No.2018WS417) and the project of Leading Talented Persons of Liaocheng.

\section{Contributions}

JLC, LJL and ZAQ contributed to conception, design, data analysis and interpretation, and are major responsible for manuscript draft and revision. JLC, LJL and ZD contributed to operation, data acquisition and patient management; LKY and ZB contributed to project coordination. All authors contributed drafting manuscript and revision, approved the submission and agreed to be accountable for this work. 


\section{Acknowledgements}

Not applicable.

\section{References}

1. Asnaashari $\mathrm{M}$, et al. Expedited removal of pyogenic granuloma by diode laser in a pediatric patient. $\mathrm{J}$ Lasers Med Sci. 2015;6(1):40-4.

2. Giblin AV, et al. Pyogenic granuloma - the quest for optimum treatment: audit of treatment of 408 cases. J Plast Reconstr Aesthet Surg. 2007;60(9):1030-5.

3. Simmons BJ, Chen L, Hu S. Pyogenic granuloma association with isotretinoin treatment for acne. Australas J Dermatol. 2016;57(4):e144-5.

4. Abaalkhail F, et al. Lobular capillary hemangioma of the liver. Hepatobiliary Pancreat Dis Int. 2009;8(3):323-5.

5. Gilmore A, Kelsberg G, Safranek S. Clinical inquiries. What's the best treatment for pyogenic granuloma? J Fam Pract. 2010;59(1):40-2.

6. Patrizi A, Gurioli C, Dika E. Pyogenic granulomas in childhood: New treatment modalities. Dermatol Ther. 2015;28(5):332.

7. Lee J, et al. Treatment options for cutaneous pyogenic granulomas: a review. J Plast Reconstr Aesthet Surg. 2011;64(9):1216-20.

8. Patrice SJ, Wiss K, Mulliken JB. Pyogenic granuloma (lobular capillary hemangioma): a clinicopathologic study of 178 cases. Pediatr Dermatol. 1991;8(4):267-76.

9. Akamatsu T, et al. Pyogenic Granuloma: A Retrospective 10-year Analysis of 82 Cases. Tokai J Exp Clin Med. 2015;40(3):110-4.

10. Koo MG, Lee SH, Han SE. Pyogenic Granuloma: A Retrospective Analysis of Cases Treated Over a 10Year. Arch Craniofac Surg. 2017;18(1):16-20.

11. Lubahn JG, Lee RK, Karp CL. Resolution of conjunctival sessile hemangioma with topical timolol. Cornea. 2014;33(1):99-100.

12. Malik M, Murphy R. A pyogenic granuloma treated with topical timolol. Br J Dermatol. 2014;171(6):1537-8.

13. Khorsand K, Maier M, Brandling-Bennett HA. Pyogenic granuloma in a 5-month-old treated with topical timolol. Pediatr Dermatol. 2015;32(1):150-1.

14. Metson R, Holmium:YAG laser endoscopic sinus surgery: a randomized, controlled study. Laryngoscope, 1996. 106(1 Pt 2 Suppl 77): p. 1-18.

15. Zhang K, Zhou Y. Effect of Pyogenic Granuloma Treated with Ultra Pulsed CO2 Laser and Liquid Nitrogen Cryosurgery. APPLIED LASER. 2013;33(2):216-8.

16. Fekrazad R, et al. Pyogenic Granuloma: Surgical Treatment with Er:YAG Laser. J Lasers Med Sci. 2014;5(4):199-205. 
17. Truschnegg A, et al. CO2 Laser Excision of a Pyogenic Granuloma Associated with Dental Implants: A Case Report and Review of the Literature. Photomed Laser Surg. 2016;34(9):425-31.

18. Hammes S, et al. Pyogenic granuloma: treatment with the 1,064-nm long-pulsed neodymium-doped yttrium aluminum garnet laser in 20 patients. Dermatol Surg. 2012;38(6):918-23.

19. Dong J, et al. Efficacy of Nd-YAG laser for treatment of pyogenic granuloma on the fingers and toes. Lasers Med Sci. 2019;34(1):41-5.

20. White JM, et al. Nd:YAG and CO2 laser therapy of oral mucosal lesions. J Clin Laser Med Surg. 1998;16(6):299-304.

21. Sutter E, et al. CO2 laser application in stomatology. Swiss Dent J. 2019;129(3):214-5.

22. Kavak A, Alper M, Aydogan I. Port-wine stain, laser therapy and pregnancy: risk factors for multiple pyogenic granulomas? J Eur Acad Dermatol Venereol. 2007;21(7):1003-4.

23. Bourguignon $R$, et al. Treatment of pyogenic granulomas with the Nd-YAG laser. J Dermatolog Treat. 2006;17(4):247-9.

\section{Tables}

Due to technical limitations, table 1 and table 2 are only available as a download in the Supplemental Files section.

\section{Figures}

Case A

Case B

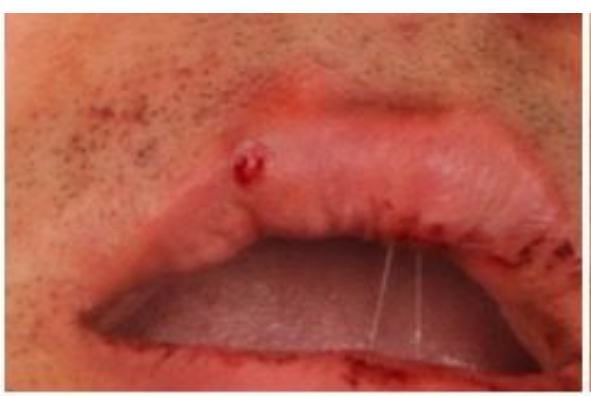

Before treatment

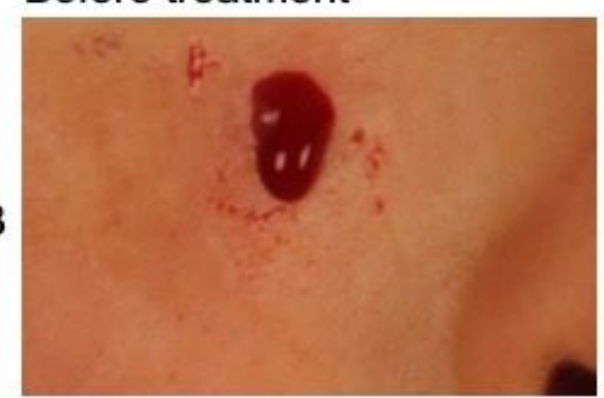

Before treatment

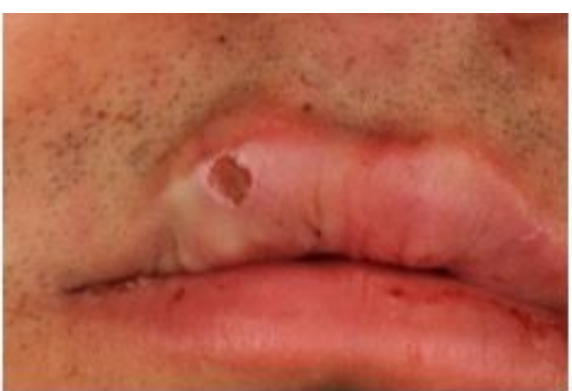

After treatment

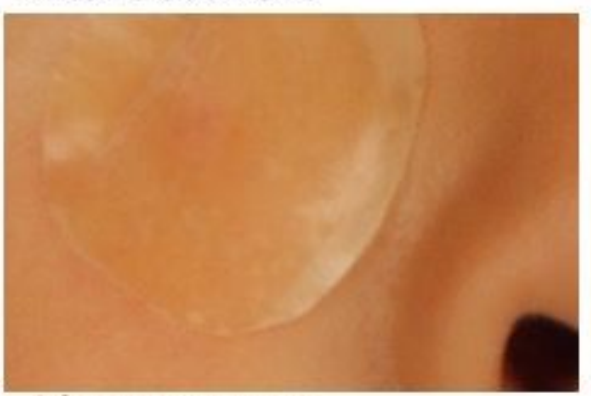

After treatment

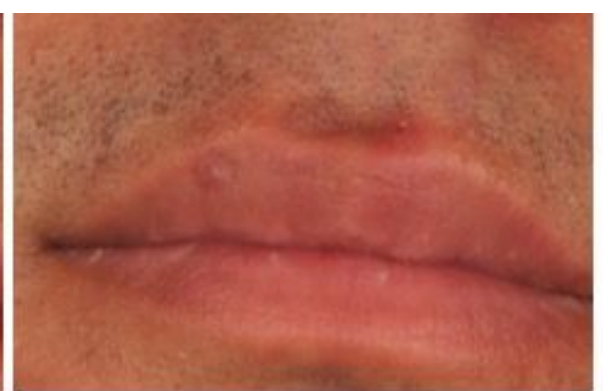

2 month after treatment

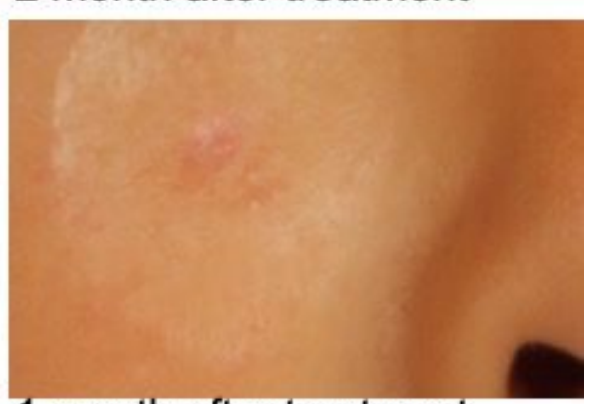

1 month after treatment

Figure 1 
Two representative cases. Two representative cases of pyogenic granuloma before and after ultrapulsed $\mathrm{CO} 2$ laser and long-pulsed Nd: YAG laser combined treatment. Photos of Case B were taken with algoplaque covered.

\section{Supplementary Files}

This is a list of supplementary files associated with this preprint. Click to download.

- Table2.xls

- Table1.xIs 